\title{
Grand challenges in pediatric endocrinology
}

\section{Stefano Cianfarani*}

Molecular Endocrinology Unit, D.P.U.O. 'Bambino Gesù' Children's Hospital and 'Rina Balducci' Center of Pediatric Endocrinology, Tor Vergata University, Rome, Italy

*Correspondance: stefano.cianfarani@uniroma2.it

Pediatric Endocrinology strives to advance the understanding and management of endocrine diseases in childhood. Although both basic and clinical research have provided important new insights into many endocrine diseases, the mechanisms underlying several clinical conditions have not yet been elucidated and many new potential therapeutic interventions remain to be tested. It has also become increasingly apparent that experiences and exposures throughout fetal life and childhood can have important effects on many diseases that develop much later during adult life. Alterations to the endocrine system mediate many of these long-term effects and understanding how these changes occur and how they translate into compromised health in adulthood have become additional questions that confront pediatric endocrinologists.

I will quote only a few examples of the grand challenges that wait to be tackled in the near future.

There is increasing evidence that the hypothalamus is involved in the regulation of energy balance and glucose homeostasis and, ultimately, in the occurrence of diabetes (Elmquist and Marcus, 2003; Prodi and Obici, 2006). In humans, however, studies on the brain control of metabolism and endocrine system are difficult and only translational research will provide robust data to be translated into clinical practice. Although the transfer of information from basic to clinical science requires hard work and is often disappointing, it is certainly worthwhile as shown by the results achieved in other disciplines such as Oncology.

For almost a century endocrine research has focused on the interaction between hormones and their receptors, providing major advances in the understanding of endocrine system physiology. More recently, the research focus has shifted to the pathways downstream from the receptor. The study of intracellular hormone signaling has revealed that some endocrine diseases may originate from alterations of signal transduction
(Kofoed et al., 2003). It is easy to foresee that further investigations will reveal that many yet unexplained endocrinopathies are the result of subtle alterations of intracellular signaling.

Novel genes have recently been discovered to play a pivotal role in the regulation of reproduction and growth (Weedon and Frayling, 2008; Bianco and Kaiser, 2009). Genome wide association analysis has revealed the influence of many unexpected genes in the control of growth, puberty and diabetes (Dateki et al., 2008; Zeggini et al., 2008; Ong et al., 2009). This new large-scale genetic approach challenges the researchers to obtain more complete descriptions of the susceptibility architecture of endocrine traits and to translate the information gathered into improvements in clinical management. However, the mechanisms by which genetic information is translated into phenotypic features and diseases as well as those underlying the interaction among hundreds of genes are still largely unknown. Genetic manipulation of experimental species, which uses transgenic and gene-knockout technology, has led and will lead to important advances in determining the relationship between genes and the function of their encoded proteins in the intact organism.

Alterations in the embryo-fetal and early postnatal hormonal environment, caused by either maternal diet or exposure to environmental factors, can modify the epigenome, and these modifications are inherited in somatic daughter cells and maintained throughout life, ultimately leading to permanent metabolic and endocrine changes (Gluckman et al., 2008). We are at the beginning of the epigenomics era that may provide important insights to be translated into interventions to revert epigenetic programming. Early prevention of adult diseases is nowadays a primary objective of Pediatrics in general and Pediatric Endocrinology in particular. Obesity, diabetes, hypertension and cardiovascular disease in adulthood may originate during embryo-fetal development and early postnatal life (Barker et al., 1989; Robinson et al., 1992; Barker, 1995). Therefore, the elucidation of environmental, (epi)genetic, and endocrine mechanisms leading to longterm metabolic risk represents a primary task of the Pediatric Endocrinologist.

The last two decades have witnessed growing concerns over the potential adverse effects that may result from exposure to a group of chemicals that have the potential to alter the normal functioning of the endocrine system in wildlife and humans. Potential adverse outcomes in both wildlife and humans have focused mainly on reproductive and sexual development and function, altered immune and nervous system, thyroid function and hormone-related cancers. Analysis of the human data by itself, while generating concerns, has so far failed to provide firm evidence of direct causal associations between exposure to endocrine disruptors and adverse health outcomes. Our current understanding of the effects posed by endocrine disruptors to humans is incomplete. Uncertainty over the possible effects of chronic exposures to a number of chemicals with endocrine disrupting potential and the fundamental role played by the endocrine system in maintaining homeostasis make the study of the effects posed by exposure to these chemicals a worldwide research priority.

Further challenges come from the development of novel therapeutic approaches to endocrine diseases in childhood. New drug formulations, individualized treatments based on pharmacogenomics, as well as gene and stem cell therapies represent further research fields for the Pediatric Endocrinologist of the 21st century.

Despite the decline in the total number of young pediatricians who are choosing subspecialties, the demands for pediatric research expertise in all disciplines, especially endocrinology, are increasing. American and European Regulatory Agencies (Food and Drug Administration, FDA, and European Medicines Agency, 
EMA) now require studies specifically designed to investigate pharmacological and toxicological aspects in pediatric populations, to test efficacy and safety and to identify the appropriate dosages and formulations. This will therefore be an increasing demand that will require the expertise of pediatric subspecialists.

\section{REFERENCES}

Barker, D. J. P. (1995). Fetal origins of coronary heart disease. Br. Med. J. 311, 171-174.

Barker, D. J. P., Winter, P. D., Osmond, C., and Margetts, B. (1989). Weight in infancy and death from ischaemic heart disease. Lancet 2271, 577-580.

Bianco, S. D., and Kaiser, U. B. (2009). The genetic and molecular basis of idiopathic hypogonadotropic hypogonadism. Nat. Rev. Endocrinol. 5, 569-576.

Dateki, S., Fukami, M., Sato, N., Muroya, K., Adachi, M., and Ogata, T. (2008). OTX2 mutation in a patient with anophthalmia, short stature, and partial growth hormone deficiency: functional studies using the IRBP, HESX1, and POU1F1 promoters. J. Clin. Endocrinol. Metab. 93, 3697-3702.
Elmquist, J. K., and Marcus, J. N. (2003). Rethinking the central causes of diabetes. Nat. Med. 9, 645-647.

Gluckman, P. D., Hanson, M. A., Cooper, C., and Thornburg, K. L. (2008). Effect of in utero and earlylife conditions on adult health and disease. $N$. Engl. J. Med. 359, 61-73.

Kofoed, E. M., Hwa, V., Little, B., Woods, K. A., Buckway, C. K., Tsubaki, J., Pratt, K. L., Bezrodnik, L., Jasper, H., Tepper, A., Heinrich, J. J., and Rosenfeld, R. G. (2003). Growth hormone insensitivity associated with a STAT5b mutation. N. Engl. J. Med. 349, 1139-1147.

Ong, K. K., Elks, C. E., Li, S., Zhao, J. H., Luan, J., Andersen, L. B., Bingham, S. A., Brage, S., Smith, G. D., Ekelund, U., Gillson, C. J., Glaser, B., Golding, J., Hardy, R., Khaw, K.-T., Kuh, D., Luben, R., Marcus, M., McGeehin, M. A., Ness, A. R., Northstone, K., Ring, S. M., Rubin, C., Sims, M. A., Song, K., Strachan, D. P., Vollenweider, P., Waeber, G., Waterworth, D. M., Wong, A., Deloukas, P, Barroso, I., Mooser, V., Loos, R. L., and Wareham, N. J. (2009). Genetic variation in LIN28B is associated with the timing of puberty. Nat. Genet. 41, 729-733.

Prodi, E., and Obici, S. (2006). The brain as a molecular target for diabetic therapy. Endocrinology 147, 2664-2669.
Robinson, R., Walton, R., Clark, P., Barker, D. J. P., Hales, C., and Osmond, C. (1992). The relation of fetal growth to plasma glucose in young man. Diabetologia $35,444-446$.

Weedon, M. N., Frayling, T. M. (2008). Reaching new heights: insights into the genetics of human stature. Trends Genet. 24, 595-603.

Zeggini, E., Scott, L. J., Saxena, R., and Voight, B. F., for the Diabetes Genetics Replication And Meta- analysis (DIAGRAM) Consortium (2008). Meta-analysis of genome-wide association data and large-scale replication identifies additional susceptibility loci for type 2 diabetes. Nat. Genet. 40, 638-645.

Received: 18 August 2010; accepted: 18 August 2010; published online: 30 September 2010.

Citation: Cianfarani S (2010) Grand challenges in pediatric endocrinology. Front. Endocrin. 1:1 doi:10.3389/ fendo.2010.00001

This article was submitted to Frontiers in Pediatric Endocrinology, a specialty of Frontiers in Endocrinology. Copyright (0) 2010 Cianfarani. This is an open-access article subject to an exclusive license agreement between the authors and the Frontiers Research Foundation, which permits unrestricted use, distribution, and reproduction in any medium, provided the original authors and source are credited. 